MATHEMATICS OF COMPUTATION

Volume 70, Number 236, Pages 1417-1436

S $0025-5718(01) 01269-8$

Article electronically published on February 8, 2001

\title{
A SPECTRAL METHOD FOR THE STOKES PROBLEM IN THREE-DIMENSIONAL UNBOUNDED DOMAINS
}

\author{
L. HALPERN
}

\begin{abstract}
We present a method for solving the Stokes problem in unbounded domains. It relies on the coupling of the transparent boundary operator and a spectral method in spherical coordinates. It is done explicitly by the use of vector-valued spherical harmonics. A uniform inf-sup condition is proved, which provides an optimal error estimate.
\end{abstract}

\section{INTRODUCTION}

When solving a steady partial differential equation in an unbounded domain, various strategies can be used. Most of them rely on the use of integral equations and fast algorithms (see for instance [15, 44, 14], and references therein). An alternative approach is to introduce an artificial boundary and a coupling between a numerical method in the interior and either an eigenmode decomposition or an integral equation on the artificial boundary ([8], [1]). Successful computations using spectral methods in two dimensions have been reported in 2], and theoretical formalism for the two-dimensional Stokes problem has been introduced in [9]. It relies on a polar decomposition of vector fields and produces an optimal method, i.e., with a uniform inf-sup condition. The aim of the present paper is to extend the method to the three-dimensional Stokes problem. We start in Section 2 with the Stokes system in a ball, with a Dirichlet boundary condition

$$
\begin{cases}-\Delta \mathbf{u}+\operatorname{grad} p=\mathbf{f} & \text { in } \Omega, \\ \operatorname{div} \mathbf{u}=0 & \text { in } \Omega, \\ \mathbf{u}=\mathbf{0} & \text { on } \Gamma .\end{cases}
$$

We write a mixed formulation in $\left(H_{0}^{1}(\Omega)\right)^{3} \times L_{0}^{2}(\Omega)$, i.e., find $(\mathbf{u}, p)$ in $X \times M$ such that

$$
\left\{\begin{array}{l}
\forall \mathbf{v} \in X, a(\mathbf{u}, \mathbf{v})+b(\mathbf{v}, p)=L(\mathbf{v}), \\
\forall q \in M, b(\mathbf{u}, q)=0
\end{array}\right.
$$

where $X$ is $\left(H_{0}^{1}(\Omega)\right)^{3}$ and $M$ is $L_{0}^{2}(\Omega)$.

Owing to a well-known result by Babuska and Brezzi (see [7]), the most delicate property to fulfill for the well-posedness is the so-called inf-sup condition. It necessitates the computation of the following quantity:

$$
\inf _{q \in M} \sup _{\mathbf{v} \in X} \frac{|b(\mathbf{v}, q)|}{\|\mathbf{v}\|_{X}\|q\|_{M}} .
$$

Received by the editor March 18, 1999 and, in revised form, October 13, 1999.

2000 Mathematics Subject Classification. Primary 35C10, 35G15, 65M70, 65 T10. 
We shall prove this quantity to be actually equal to $\frac{1}{\sqrt{3}}$. The result is achieved by a convenient decomposition of vector fields in subsection 2.3. It relies on the use of the eigenmodes for the vector-valued Laplace-Beltrami operator on the sphere. These eigenmodes are the vector-valued spherical harmonics (see [14). They have the remarkable ability to diagonalize the Stokes operator. This allows for an explicit resolution of the Stokes problem in subsection 2.4

Given such a decomposition in a ball, it is now easy to introduce a Galerkin method in Section 3 we use "almost" polynomials in the radial variable and a finite number of spherical harmonics in a consistent way (see subsections 3.1 and [3.2). This process creates spaces $X_{N}$ and $M_{N}$ in which we solve (1.2). The discrete spaces are such that the inf-sup number is still equal to $\frac{1}{\sqrt{3}}$. According to classical theory, this property prevents the apparition of parasitic modes for the pressure and allows for optimal error estimates, as proved in subsection 3.3

With all these tools in hand, we are now able to handle the problem of unbounded domains in Section 4 . Considering the Stokes problem in $\mathbb{R}^{3}$, we show how to reduce the problem to a bounded domain by introducing a fictitious boundary, the sphere of radius $R$, in subsection 4.1 In subsection 4.2. we give an explicit representation of the transparent operator, which in turn provides easy proofs of its properties. The last two subsections in Section 4 are devoted to a Galerkin method for the problem. The path is the same as in Sections 2 and 3 and gives the exact value in the inf-sup condition.

\section{The Stokes System in a BALL}

2.1. A few elements in functional analysis. We recall here some well-known results on Sobolev spaces. For details see [7].

For any three-dimensional domain $\Omega, L^{2}(\Omega)$ is the (complex) Hilbert space of square-integrable functions, furnished with the inner product

$$
(v, w)_{0, \Omega}=\int_{\Omega} v(x) \overline{w(x)} d x
$$

and the corresponding norm is denoted by $\|\cdot\|_{0, \Omega}$. $\mathcal{D}(\Omega)$ is the space of functions which are infinitely differentiable and compactly supported in $\Omega$. Its dual space is the space of distributions, called $\mathcal{D}^{\prime}(\Omega)$. For any positive integer $m, H^{m}(\Omega)$ is the Sobolev space of distributions defined in $\Omega$, whose derivatives up to order $m$ belong to $L^{2}(\Omega)$, furnished with the inner product

$$
(v, w)_{m, \Omega}=\sum_{|k| \leq m}\left(D^{k} v, D^{k} w\right)_{0, \Omega}
$$

and the corresponding norm is denoted by $\|\cdot\|_{m, \Omega}$ (as usual, $H^{0}(\Omega)$ is identical to $L^{2}(\Omega)$ ). The closure of $\mathcal{D}(\Omega)$ in $H^{1}(\Omega)$ is denoted by $H_{0}^{1}(\Omega)$, and $H^{-1}(\Omega)$ is the dual space of $H_{0}^{1}(\Omega)$. The duality between $H^{-1}(\Omega)$ and $H_{0}^{1}(\Omega)$ is denoted by $\langle.,$.$\rangle .$

If $\Omega$ is bounded with smooth boundary $\Gamma, H_{0}^{1}(\Omega)$ is the set of functions in $H^{1}(\Omega)$ which vanish on $\Gamma$. Then, due to the Poincaré-Friedrichs inequality, the semi-norm defined on $H^{1}(\Omega)$ by $|v|_{1, \Omega}=\|\operatorname{grad} v\|_{0, \Omega}$ is a norm on $H_{0}^{1}(\Omega)$, equivalent to the $\|\cdot\|_{1, \Omega}$-norm. The space of traces on $\Gamma$ of the elements of $H^{1}(\Omega)$ is called $H^{1 / 2}(\Gamma)$, and the dual space is $H^{-1 / 2}(\Gamma)$. The duality between $H^{1 / 2}(\Gamma)$ and $H^{-1 / 2}(\Gamma)$ is denoted by $\langle., .\rangle_{\Gamma} . L_{0}^{2}(\Omega)$ is the space of elements $q$ in $L^{2}(\Omega)$ such that $\int_{\Omega} q(x) d x=$ 0 . For simplicity, for any $m \geq-1$, we shall call $\mathbf{H}^{m}(\Omega)$ the space $\left(H^{m}(\Omega)\right)^{3}$ (resp. $\left.\mathbf{L}^{2}(\Omega)=\left(L^{2}(\Omega)\right)^{3}\right)$. The notations $(., .)_{m, \Omega}$ and $\|\cdot\|_{m, \Omega}$ apply indiscriminately to 
$H^{m}(\Omega)$ or $\mathbf{H}^{m}(\Omega)$. Furthermore, in the following any notation could be vectorized by boldfacing.

In order to analyze the Stokes problem in bounded or exterior domains, with various boundary conditions, it is convenient to introduce the following abstract frame (see [7). Let $M$ and $X$ be two Hilbert spaces, furnished with the inner product $(., .)_{M}$ and $(., .)_{X}$, and the corresponding norms $\|.\|_{M}$ and $\|.\|_{X}$. Let $a$ be a sesquilinear form on $X \times X$ (i.e., linear with respect to the first variable and antilinear with respect to the second variable) and $b$ be a sesquilinear form on $X \times M$ (i.e., linear with respect to the variable in $M$ and antilinear with respect to the variable in $X)$. We shall call the mixed or saddle point formulation the following problem. Find $(\mathbf{u}, p)$ in $X \times M$ such that

$$
\left\{\begin{array}{l}
\forall \mathbf{v} \in X, a(\mathbf{u}, \mathbf{v})+b(\mathbf{v}, p)=L(\mathbf{v}) \\
\forall q \in M, b(\mathbf{u}, q)=0
\end{array}\right.
$$

We introduce the subspace of $X, V=\{\mathbf{v} \in X, \forall q \in M, b(\mathbf{v}, q)=0\}$. The general result by Babuska and Brezzi reads (see 7])

Theorem 2.1. Suppose the following assumptions are fulfilled:

1. The sesquilinear form a is continuous on $X$ and there exists a positive constant $\alpha$ such that for any $\mathbf{v}$ in $V$,

$$
a(\mathbf{v}, \mathbf{v}) \geq \alpha\|\mathbf{v}\|_{X}^{2}
$$

2. The sesquilinear form $b$ is continuous on $X \times M$.

3. The inf-sup condition is fulfilled: there exists a real number $C$ positive such that

$$
\inf _{q \in M} \sup _{\mathbf{v} \in X} \frac{|b(\mathbf{v}, q)|}{\|\mathbf{v}\|_{X}\|q\|_{M}} \geq C
$$

4. $L$ is an antilinear continuous form on $X$.

Then problem (2.3) has a unique solution.

2.2. Variational formulation in a bounded domain. Let $\Omega$ be a bounded open connected subset of $\mathbb{R}^{3}$ with smooth boundary $\Gamma$. The Stokes problem in $\Omega$ with homogeneous Dirichlet boundary data reads: Find $(\mathbf{u}, p)$ such that

$$
\begin{cases}-\Delta \mathbf{u}+\operatorname{grad} p=\mathbf{f} & \text { in } \Omega \\ \operatorname{div} \mathbf{u}=0 & \text { in } \Omega \\ \mathbf{u}=\mathbf{0} & \text { on } \Gamma\end{cases}
$$

The notations grad, div and $\Delta$ give the gradient, divergence and Laplace operators, respectively,

$$
\operatorname{grad} p=\left(\frac{\partial p}{\partial x_{1}}, \frac{\partial p}{\partial x_{2}}, \frac{\partial p}{\partial x_{3}}\right), \quad \operatorname{div} \mathbf{u}=\sum_{i=1}^{3} \frac{\partial u_{i}}{\partial x_{i}}, \quad \Delta \varphi=\sum_{i=1}^{3} \frac{\partial^{2} \varphi}{\partial x_{i}^{2}} .
$$


Problem (2.6) enters in the previous frame by defining $X=\mathbf{H}_{0}^{1}(\Omega)$ provided with the semi-norm $|\cdot|_{1, \Omega}$ and $M=L_{0}^{2}(\Omega)$. The forms $a$ and $b$ are given by

$$
\left\{\begin{array}{l}
a(\mathbf{u}, \mathbf{v})=\sum_{(i, j)=1}^{3}\left(\frac{\partial u_{i}}{\partial x_{j}}, \frac{\partial v_{i}}{\partial x_{j}}\right)_{0, \Omega}, \\
b(\mathbf{v}, q)=-(q, \operatorname{div} \mathbf{v})_{0, \Omega}, \\
L(\mathbf{v})=\langle\mathbf{f}, \mathbf{v}\rangle .
\end{array}\right.
$$

We shall call a solution to (2.3) with the definitions in (2.8) a weak solution to (2.6). Theorem 2.1] gives the well-posedness of problem (2.6) as follows (see [7]):

Theorem 2.2. For any $\mathbf{f}$ in $\mathbf{H}^{-1}(\Omega)$, there exists a unique weak solution $(\mathbf{u}, p)$ to (2.6) in $\mathbf{H}_{0}^{1}(\Omega) \times L_{0}^{2}(\Omega)$. Furthermore, there exists a positive constant $C$ such that

$$
\forall \mathbf{f} \in \mathbf{H}^{-1}(\Omega),\|\mathbf{u}\|_{1, \Omega}+\|p\|_{0, \Omega} \leq C\|\mathbf{f}\|_{-1, \Omega} .
$$

Moreover if $\mathbf{f}$ belongs to $\mathbf{H}^{m}(\Omega)$, then $(\mathbf{u}, p)$ belongs to $\mathbf{H}^{m+2}(\Omega) \times \mathbf{H}^{m+1}(\Omega)$ and there exists a positive constant $D$ such that

$$
\forall \mathbf{f} \in \mathbf{H}^{m}(\Omega),\|\mathbf{u}\|_{m+2, \Omega}+\|p\|_{m+1, \Omega} \leq D\|\mathbf{f}\|_{m, \Omega}
$$

We shall now consider the case where $\Omega$ is the unit ball. We shall identify the best constant in the inf-sup condition. For that purpose, we introduce a decomposition of vector fields on the sphere.

2.3. Spherical harmonics, vector-valued spherical harmonics. We describe here a theory based on well-known results in quantum mechanics (see [3]). They have been introduced in the frame of functional analysis by Nedelec to handle exterior problems for Maxwell equations ([14]).

A point in $\mathbb{R}^{3}$ is defined by its spherical coordinates $(r, \theta, \varphi)$, and the corresponding moving frame is $\left\{\mathbf{e}_{r}, \mathbf{e}_{\theta}, \mathbf{e}_{\varphi}\right\}$. These coordinates are related to the euclidean coordinates $\left\{x_{1}, x_{2}, x_{3}\right\}$ by

$$
\left\{\begin{array}{l}
x_{1}=r \sin \theta \cos \varphi \\
x_{2}=r \sin \theta \sin \varphi, \\
x_{3}=r \cos \theta .
\end{array}\right.
$$

On $S$, sphere in $\mathbb{R}^{3}$ of radius $1, L^{2}(S)$ is the (complex) Hilbert space of squareintegrable functions on $S$, furnished with the inner product

$$
(v, w)_{0, S}=\int_{S} v(s) \overline{w(s)} d s=\int_{0}^{2 \pi} \int_{0}^{\pi} v(\theta, \varphi) \overline{w(\theta, \varphi)} \sin \theta d \theta d \varphi
$$

and the corresponding norm is denoted by $\|\cdot\|_{0, S}$.

The Laplace-Beltrami operator $\Delta_{S}$ is given by

$$
\Delta_{S}=\frac{1}{\sin ^{2} \theta} \frac{\partial^{2}}{\partial \varphi^{2}}+\frac{1}{\sin \theta} \frac{\partial}{\partial \theta}\left(\sin \theta \frac{\partial}{\partial \theta}\right) .
$$

$-\Delta_{S}$ is a self-adjoint positive compact operator in $L^{2}(S)$. Thus there is a complete set of eigenfunctions in $L^{2}(S)$. The eigenvalues are $m(m+1)$ for $m \geq 0$. For any $m \geq 0$, the corresponding eigenspace $\mathcal{Y}_{m}$ has dimension $2 m+1$. A classical basis, orthonormal in $L^{2}(S)$, is denoted by $\left\{Y_{m, l}\right\}_{-m \leq l \leq m}$. They satisfy the 


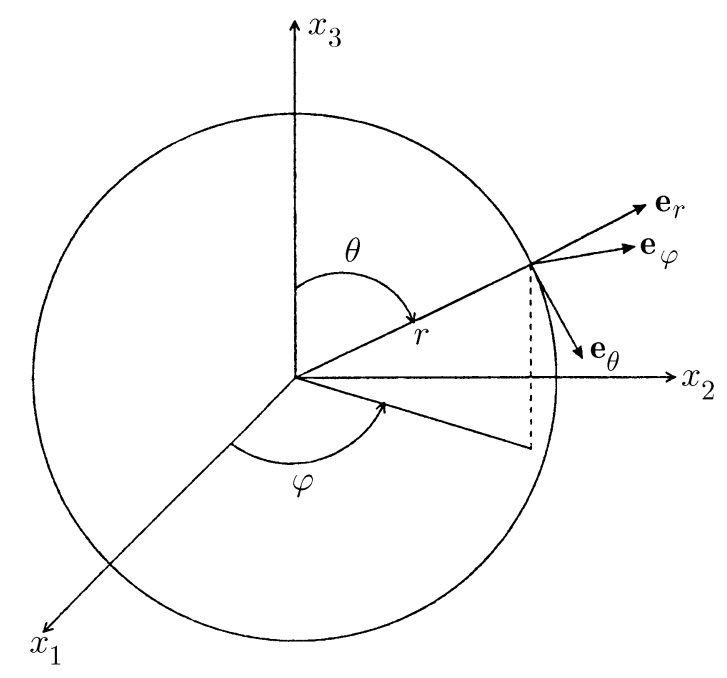

FIGURE 1. Spherical coordinates

relations

$$
\left\{\begin{array}{l}
\Delta_{S} Y_{m, l}+m(m+1) Y_{m, l}=0, m \geq 0,-m \leq l \leq m, \\
\left(Y_{m, l}, Y_{p, k}\right)_{0, S}= \begin{cases}1 & \text { if } m=p \text { and } l=k \\
0 & \text { otherwise }\end{cases}
\end{array}\right.
$$

The functions $Y_{m, l}$ are written in closed form as

$$
Y_{m, l}(\theta, \varphi)=(-1)^{l}(i)^{m}\left(\frac{m+\frac{1}{2}}{2 \pi} \frac{(m-l) !}{(m+l) !}\right)^{1 / 2} e^{i l \varphi} P_{m}^{l}(\cos \theta),
$$

where $P_{m}^{l}$ is the associate Legendre polynomial defined by

$$
\left\{\begin{array}{l}
0 \leq l \leq m, P_{m}^{l}(\cos \theta)=(\sin \theta)^{l}\left(\frac{d^{l}}{d x^{l}} P_{m}\right)(\cos \theta), \\
-m \leq l \leq 0, P_{m}^{l}(\cos \theta)=(-1)^{l} \frac{(m+l) !}{(m-l) !} P_{m}^{-l}(\cos \theta),
\end{array}\right.
$$

and $P_{m}$ is the $m$ th Legendre polynomial on $[-1,1]$ (see [1]).

We introduce the tangent gradient

$$
\operatorname{grad}_{S}=\frac{1}{\sin \theta} \frac{\partial}{\partial \varphi} \mathbf{e}_{\varphi}+\frac{\partial}{\partial \theta} \mathbf{e}_{\theta}
$$

and the Hilbert space

$$
H^{1}(S)=\left\{u \in L^{2}(S), \operatorname{grad}_{S} u \in\left(L^{2}(S)\right)^{3}\right\}
$$

furnished with the inner product

$$
(u, v)_{1, S}=(u, v)_{0, S}+\left(\operatorname{grad}_{S} u, \operatorname{grad}_{S} v\right)_{0, S} .
$$

The following Green's formula holds:

$$
\left(-\Delta_{S} u, v\right)_{0, S}=\left(\operatorname{grad}_{S} u, \operatorname{grad}_{S} v\right)_{0, S} .
$$


Thus, $\left\{Y_{m, l}\right\}_{-m \leq l \leq m, m \geq 0}$ is a unitary basis in $L^{2}(S)$, orthogonal in $H^{1}(S)$, and

$$
\left\|\operatorname{grad}_{S} Y_{m, l}\right\|_{0, S}^{2}=m(m+1) \text {. }
$$

Remark 2.1. For each $m, \mathcal{Y}_{m}$ is the space of the restriction to $S$ of the harmonic polynomials of degree $m$, i.e.,

$$
\Delta\left(r^{m} Y_{m, l}(\theta, \varphi)\right)=0 .
$$

The $Y_{m, l}$ are called the spherical harmonics.

For further purpose, following [14], we shall define, for any real number $s, H^{s}(S)$ as

$$
H^{s}(S)=\left\{g=\sum_{m=0}^{+\infty} \sum_{l=-m}^{m} g_{m, l} Y_{m, l}(\theta, \varphi), \sum_{m=0}^{+\infty} \sum_{l=-m}^{m}(m+1)^{2 s}\left|g_{m, l}\right|^{2}<+\infty\right\} .
$$

It is furnished with the norm

$$
\|g\|_{s, S}^{2}=\sum_{m=0}^{+\infty} \sum_{l=-m}^{m}(m+1)^{2 s}\left|g_{m, l}\right|^{2} .
$$

Let us come now to the vector fields. As mentioned in subsection 2.3 , $\mathbf{L}^{2}(S)$ denotes the space of vectors whose three components (in the canonical frame) belong to $L^{2}(S)$. The vector-valued Laplace-Beltrami operator is defined by

$$
\Delta_{S} \mathbf{A}=\frac{1}{\sin ^{2} \theta} \frac{\partial^{2}}{\partial \varphi^{2}}(\mathbf{A})+\frac{1}{\sin \theta} \frac{\partial}{\partial \theta}\left(\sin \theta \frac{\partial}{\partial \theta}(\mathbf{A})\right),
$$

or, to be more precise,

$$
\Delta_{S} \mathbf{A}=\left(\Delta_{S} A_{1}\right) \mathbf{e}_{1}+\left(\Delta_{S} A_{2}\right) \mathbf{e}_{2}+\left(\Delta_{S} A_{3}\right) \mathbf{e}_{3} .
$$

We can write the Green formula as

$$
\left(-\Delta_{S} \mathbf{A}, \mathbf{B}\right)_{0, S}=\left(\frac{\partial \mathbf{A}}{\partial \theta}, \frac{\partial \mathbf{B}}{\partial \theta}\right)_{0, S}+\left(\frac{1}{\sin \theta} \frac{\partial \mathbf{A}}{\partial \varphi}, \frac{1}{\sin \theta} \frac{\partial \mathbf{B}}{\partial \varphi}\right)_{0, S} .
$$

Let us now define a complete set of vectors, orthogonal in $\mathbf{L}^{2}(S)$, by

$$
\left\{\begin{array}{c}
m \geq 1,-m \leq l \leq m, \mathbf{T}_{m, l}(\theta, \varphi)=\operatorname{grad}_{S} Y_{m, l} \wedge \mathbf{e}_{r} \\
\quad=\frac{1}{\sin \theta} \frac{\partial Y_{m, l}}{\partial \varphi} \mathbf{e}_{\theta}-\frac{\partial Y_{m, l}}{\partial \theta} \mathbf{e}_{\varphi}, \\
m \geq 0,-m-1 \leq l \leq m+1, \mathbf{V}_{m, l}(\theta, \varphi)=(m+1) Y_{m+1, l} \mathbf{e}_{r}+\operatorname{grad}_{S} Y_{m+1, l} \\
m \geq 1,-m+1 \leq l \leq m-1, \mathbf{W}_{m, l}(\theta, \varphi)=m Y_{m-1, l} \mathbf{e}_{r}-\operatorname{grad}_{S} Y_{m-1, l}
\end{array}\right.
$$

They are a complete basis of eigenfunctions for the vector-valued Laplace-Beltrami operator, i.e., they satisfy the identity

$$
\Delta_{S} \mathbf{A}+m(m+1) \mathbf{A}=0
$$

and their norms are given by

$$
\left\|\mathbf{T}_{m, l}\right\|_{0, S}^{2}=m(m+1),\left\|\mathbf{V}_{m, l}\right\|_{0, S}^{2}=(2 m+3)(m+1),\left\|\mathbf{W}_{m, l}\right\|_{0, S}^{2}=m(2 m-1) .
$$

Remark 2.2. The set of vectors in (2.27) are the vector-valued spherical harmonics, i.e., the traces on $S$ of vectors whose three components are harmonic polynomials. 
Remark 2.3. There is another vector-valued Laplace-Beltrami operator, defined on the tangent space $T L^{2}(S)$ by

$$
\tilde{\Delta}_{S}=\operatorname{grad}_{S}\left(\operatorname{div}{ }_{S}\right)-\operatorname{curl}_{S}\left(\operatorname{curl}_{S}\right),
$$

where, for $u=u(\theta, \varphi)$, the vector-valued curl operator is given by

$$
\operatorname{curl}_{S} u=-\frac{\partial u}{\partial \theta} \mathbf{e}_{\varphi}+\frac{1}{\sin \theta} \frac{\partial u}{\partial \varphi} \mathbf{e}_{\theta}
$$

and, for $\mathbf{A}(\theta, \varphi)=A_{\theta}(\theta, \varphi) \mathbf{e}_{\theta}+A_{\varphi}(\theta, \varphi) \mathbf{e}_{\varphi}$, the divergence and scalar curl operators are defined by

$$
\begin{aligned}
\operatorname{div}_{S} \mathbf{A} & =\frac{1}{\sin \theta}\left(\frac{\partial}{\partial \theta}\left(\sin \theta A_{\theta}\right)+\frac{\partial}{\partial \varphi}\left(A_{\varphi}\right)\right), \\
\operatorname{curl}_{S} \mathbf{A} & =\frac{1}{\sin \theta}\left(\frac{\partial}{\partial \theta}\left(\sin \theta A_{\varphi}\right)-\frac{\partial}{\partial \varphi}\left(A_{\theta}\right)\right) .
\end{aligned}
$$

This operator has the same properties as $\Delta_{S}$ : it is self-adjoint, negative on $T L^{2}(S)$. For any $\mathbf{A}$ in $L^{2}(S)$, the projection of $\Delta_{S} \mathbf{A}$ on the tangent space vect $\left\{\mathbf{e}_{\theta}, \mathbf{e}_{\varphi}\right\}$ coincides with $\tilde{\Delta}_{S} \mathbf{A}^{T}$, where $\mathbf{A}^{T}$ is the projection of $\mathbf{A}$ on vect $\left\{\mathbf{e}_{\theta}, \mathbf{e}_{\varphi}\right\}$. The Laplace-Beltrami operator we defined first is better suited for our purpose.

Thus, any $p$ in $L^{2}(\Omega)$ can be expanded as

$$
p=\sum_{m=0}^{+\infty} \sum_{l=-m}^{m} p_{m, l}(r) Y_{m, l}(\theta, \varphi) .
$$

By separation of variables, the following space of functions depending on the $r$ variable arises naturally:

$$
L_{r^{2}}^{2}=L_{r^{2}}^{2}(] 0,1[)=\left\{\varphi \in \mathcal{D}^{\prime}(] 0,1[), \int_{0}^{1} r^{2}|\varphi|^{2}(r) d r<+\infty\right\} .
$$

It is a Hilbert space when equipped with the natural inner product

$$
(\varphi, \psi)_{r}=\int_{0}^{1} r^{2} \varphi(r) \overline{\psi(r)} d r
$$

and the corresponding norm, denoted by $\|\cdot\|_{r}$.

The Parseval identity reads in this case

$$
\|p\|_{0, \Omega}^{2}=\sum_{m=0}^{+\infty} \sum_{l=-m}^{m}\left\|p_{m, l}\right\|_{r}^{2} .
$$

Any $\mathbf{u}$ in $\mathbf{L}^{2}(\Omega)$ can be expanded in the same was as

$$
\begin{aligned}
\mathbf{u}= & \sum_{m=1}^{+\infty} \sum_{l=-m}^{m} t_{m, l}(r) \mathbf{T}_{m, l}(\theta, \varphi) \\
& +\sum_{m=0}^{+\infty} \sum_{l=-m-1}^{m+1} v_{m, l}(r) \mathbf{V}_{m, l}(\theta, \varphi)+\sum_{m=1}^{+\infty} \sum_{l=-m+1}^{m-1} w_{m, l}(r) \mathbf{W}_{m, l}(\theta, \varphi),
\end{aligned}
$$


and the Parseval identity reads

$$
\begin{aligned}
\|\mathbf{u}\|_{0, \Omega}^{2}= & \sum_{m=1}^{+\infty} \sum_{l=-m}^{m} m(m+1)\left\|t_{m, l}\right\|_{r}^{2} \\
& +\sum_{m=0}^{+\infty} \sum_{l=-m-1}^{m+1}(m+1)(2 m+3)\left\|v_{m, l}\right\|_{r}^{2}+\sum_{m=1}^{+\infty} \sum_{l=-m+1}^{m-1} m(2 m-1)\left\|w_{m, l}\right\|_{r}^{2} .
\end{aligned}
$$

The scalar product $a(.,$.$) defined in (2.8) can be expressed in spherical coordi-$ nates as

$$
a(\mathbf{u}, \mathbf{v})=\left(\frac{\partial \mathbf{u}}{\partial r}, \frac{\partial \mathbf{v}}{\partial r}\right)_{0, \Omega}+\left(\frac{1}{r} \frac{\partial \mathbf{u}}{\partial \theta}, \frac{1}{r} \frac{\partial \mathbf{v}}{\partial \theta}\right)_{0, \Omega}+\left(\frac{1}{r \sin \theta} \frac{\partial \mathbf{u}}{\partial \varphi}, \frac{1}{r \sin \theta} \frac{\partial \mathbf{v}}{\partial \varphi}\right)_{0, \Omega} .
$$

If $\mathbf{u}$ and $\mathbf{v}$ are written as in (2.37) with coefficients $\left(t_{m, l}, v_{m, l}, w_{m, l}\right)$ and $\left(\tilde{t}_{m, l}, \tilde{v}_{m, l}, \tilde{w}_{m, l}\right)$, respectively, then $a(\mathbf{u}, \mathbf{v})$ can be expressed as

$$
\begin{aligned}
a(\mathbf{u}, \mathbf{v})= & \sum_{m=1}^{+\infty} \sum_{l=-m}^{m} m(m+1)\left(\left(t_{m, l}^{\prime}, \tilde{t}_{m, l}^{\prime}\right)_{r}+m(m+1)\left(\frac{t_{m, l}}{r}, \frac{\tilde{t}_{m, l}}{r}\right)_{r}\right) \\
& +\sum_{m=0}^{+\infty} \sum_{l=-m-1}^{m+1}(m+1)(2 m+3)\left(\left(v_{m, l}^{\prime}, \tilde{v}_{m, l}^{\prime}\right)_{r}+m(m+1)\left(\frac{v_{m, l}}{r}, \frac{\tilde{v}_{m, l}}{r}\right)_{r}\right) \\
& +\sum_{m=1}^{+\infty} \sum_{l=-m+1}^{m-1} m(2 m-1)\left(\left(w_{m, l}^{\prime}, \tilde{w}_{m, l}^{\prime}\right)_{r}+m(m+1)\left(\frac{w_{m, l}}{r}, \frac{\tilde{w}_{m, l}}{r}\right)_{r}\right),
\end{aligned}
$$

where the sign ' denotes the derivative with respect to the variable $r$. Assume now that $\mathbf{u}$ and $\mathbf{v}$ belong to $\mathbf{H}_{0}^{1}(\Omega)$. Then all the $r$-variables vanish for $r=1$. Furthermore, if $g$ denotes any of these functions, $g, \frac{g}{r}, g^{\prime}$ belong to $L_{r^{2}}^{2}$. Then, according to [13], $r^{\frac{1}{2}} g(0)$ can be defined in a weak sense and the value is 0 . Thus, introducing the new functions

$$
\left\{\begin{array}{l}
\tau_{m, l}=t_{m, l}^{\prime}+\frac{m+1}{r} t_{m, l}, \\
\varphi_{m, l}=v_{m, l}^{\prime}-\frac{m}{r} v_{m, l}, \\
\psi_{m, l}=w_{m, l}^{\prime}+\frac{m+1}{r} w_{m, l},
\end{array}\right.
$$

we can integrate by parts in $(2.40)$ and get

$$
\begin{aligned}
a(\mathbf{u}, \mathbf{v})= & \sum_{m=1}^{+\infty} \sum_{l=-m}^{m} m(m+1)\left(\tau_{m, l}, \tilde{\tau}_{m, l}\right)_{r} \\
& +\sum_{m=0}^{+\infty} \sum_{l=-m-1}^{m+1}(m+1)(2 m+3)\left(\varphi_{m, l}, \tilde{\varphi}_{m, l}\right)_{r} \\
& +\sum_{m=1}^{+\infty} \sum_{l=-m+1}^{m-1} m(2 m-1)\left(\psi_{m, l}, \tilde{\psi}_{m, l}\right)_{r} .
\end{aligned}
$$


In particular, the norm in $X$ is given by

$$
\begin{aligned}
\|\mathbf{u}\|_{X}^{2}= & \sum_{m=1}^{+\infty} \sum_{l=-m}^{m} m(m+1)\left\|\tau_{m, l}\right\|_{r}^{2} \\
& +\sum_{m=0}^{+\infty} \sum_{l=-m-1}^{m+1}(m+1)(2 m+3)\left\|\varphi_{m, l}\right\|_{r}^{2} \\
& +\sum_{m=1}^{+\infty} \sum_{l=-m+1}^{m-1} m(2 m-1)\left\|\psi_{m, l}\right\|_{r}^{2} .
\end{aligned}
$$

\subsection{The inf-sup condition in $B_{1}$.}

Theorem 2.3. When $\Omega$ is the ball of radius 1 , one has

$$
\inf _{q \in M} \sup _{\mathbf{v} \in X} \frac{|b(\mathbf{v}, q)|}{\|\mathbf{v}\|_{X}\|q\|_{M}}=\frac{1}{\sqrt{3}}
$$

Proof. As in 9], we write

$$
\inf _{q \in M} \sup _{\mathbf{v} \in X} \frac{|b(\mathbf{v}, q)|}{\|\mathbf{v}\| X\|q\|_{M}}=\inf _{q \in M} \frac{\|\mathbf{w}(q)\|_{X}}{\|q\|_{M}},
$$

where, for $q$ in $M, \mathbf{w}(q)$ is the unique solution to the problem

$$
\mathbf{w} \in X, \forall \mathbf{v} \in X, a(\mathbf{w}, \mathbf{v})=b(\mathbf{v}, q)
$$

or equivalently,

$$
\left\{\begin{array}{l}
\mathbf{w} \in X=\mathbf{H}_{0}^{1}(\Omega) \\
\Delta \mathbf{w}+\operatorname{grad} q=\mathbf{0} .
\end{array}\right.
$$

We now expand $q$ as in (2.33) and $\mathbf{w}$ as in (2.37). $\Delta \mathbf{w}$ and $\operatorname{grad} q$ are expanded on the $\left\{\left\{\mathbf{T}_{m, l}\right\},\left\{\mathbf{V}_{m, l}\right\},\left\{\mathbf{W}_{m, l}\right\}\right\}$ basis as well, and identifying the coefficients to zero yields

$$
\forall m \geq 0, \forall l,-m-1 \leq l \leq m+1, \frac{d}{d r}\left(r^{m+2}\left(\varphi_{m, l}+\frac{1}{2 m+3} p_{m+1, l}\right)\right)=0,
$$

$$
\forall m \geq 1, \forall l,-m+1 \leq l \leq m-1, \frac{d}{d r}\left(r^{-(m-1)}\left(\psi_{m, l}+\frac{1}{2 m-1} p_{m-1, l}\right)\right)=0,
$$

where, in (2.48), $\Delta_{r}$ denotes the part of the Laplace operator corresponding to the $r$-variable, i.e.,

$$
\Delta=\Delta_{r}+\frac{1}{r^{2}} \Delta_{S}, \Delta_{r}=\frac{1}{r^{2}} \frac{\partial}{\partial r}\left(r^{2} \frac{\partial}{\partial r}\right) .
$$

In (2.49) and 2.50), the following identity has been used:

$$
\Delta_{r}=\left(\frac{d}{d r}+\frac{m+2}{r}\right)\left(\frac{d}{d r}-\frac{m}{r}\right)=\left(\frac{d}{d r}-\frac{m-1}{r}\right)\left(\frac{d}{d r}+\frac{m+1}{r}\right) .
$$


Let us start with (2.48). It can be solved explicitly as $t_{m, l}=\alpha r^{m}$. The regularity assumption, together with the boundary conditions, imply

$$
\forall m \geq 1, \forall l,-m \leq l \leq m, t_{m, l}=0 .
$$

As for (2.49), by the regularity assumptions we get

$$
\forall m \geq 1, \forall l,-m \leq l \leq m, q_{m, l}=-(2 m+1) \varphi_{m-1, l} .
$$

The previous formula (2.53) gives all the coefficients of $q$, but $q_{0,0}$, in terms of the coefficients of $\mathbf{w}$. Since $q$ belongs to $L_{0}^{2}(\Omega), \int_{0}^{1} r^{2} q_{0,0}(r) d r=0$. Writing (2.50) for $m=1$ and using the boundary condition gives

$$
q_{0,0}=-\psi_{1,0}
$$

Collecting (2.53) and (2.54) gives the upper bound

$$
\|q\|_{0, \Omega}^{2} \leq 3\|\mathbf{w}\|_{X}^{2} .
$$

Furthermore, choosing $q_{m, l}=0$ for $m \neq 1$ gives the equality in the previous formula. To summarize, for all $q$ in $L_{0}^{2}(\Omega)$, given by its coefficients $q_{m, l}$, we have found a solution $\mathbf{w}$ to (2.46). Its coefficients in the expansion (2.37) are given by

$$
\left\{\begin{array}{l}
\forall m \geq 1, \forall l,-m \leq l \leq m, t_{m, l}=0 ; \\
\forall m \geq 0, \forall l,-m-1 \leq l \leq m+1, \\
\quad v_{m, l}=\frac{1}{2 m+3} r^{m} \int_{r}^{1} \varrho^{-m} q_{m+1, l}(\varrho) d \varrho ; \\
\forall m \geq 1, \forall l,-m+1 \leq l \leq m-1, \\
w_{m, l}=\frac{1}{2 m-1}\left\{r^{m} \int_{0}^{1} \varrho^{m+1} q_{m-1, l}(\varrho) d \varrho-r^{-(m+1)} \int_{0}^{r} \varrho^{m+1} q_{m-1, l}(\varrho) d \varrho\right\} .
\end{array}\right.
$$

\section{A Galerkin method for the Stokes system in a Ball}

For any integer $L, \mathbb{P}_{L}([0,1])$ denotes the space of polynomials in the variable $r$ of degree lower than or equal to $L . \tilde{\mathbb{P}}_{L}([0,1])$ is the subspace of $\mathbb{P}_{L}([0,1])$, whose elements vanish for $r=1$. We introduce discrete spaces for pressure and velocity:

$$
\begin{aligned}
& M_{K, L}=\left\{p=\sum_{m=0}^{K-1} \sum_{l=-m}^{m} p_{m, l} Y_{m, l}, p_{m, l} \in \mathbb{P}_{L}([0,1])\right\}, \\
& X_{N, N}=\left\{\mathbf{u}=\sum_{m=1}^{N} \sum_{l=-m}^{m} t_{m, l} \mathbf{T}_{m, l}\right. \\
&+\sum_{m=0}^{N-2} \sum_{l=-m-1}^{m+1} v_{m, l} \mathbf{V}_{m, l}+\sum_{m=1}^{N} \sum_{l=-m+1}^{m-1} w_{m, l} \mathbf{W}_{m, l}, \\
&\left.t_{m, l}, w_{m, l} \in \tilde{\mathbb{P}}_{N}([0,1]), v_{m, l} \in \tilde{\mathbb{P}}_{N}([0,1]) \oplus \mathbb{Q}_{m}\right\},
\end{aligned}
$$

where

$$
\mathbb{Q}_{m}=\left\{g(r): g(r)=c r^{m} \ln r, c \in \mathbb{C}\right\} .
$$


For any integer $N \geq 2$, define

$$
\begin{gathered}
M_{N}=M \cap M_{N, N-1}, \\
X_{N}=X \cap X_{N, N} .
\end{gathered}
$$

$X_{N}$ and $M_{N}$ are furnished with the inner products of $X$ and $M$. The discrete problem is the following: find $\left(\mathbf{u}_{N}, p_{N}\right)$ in $X_{N} \times M_{N}$ such that

$$
\left\{\begin{array}{l}
\forall \mathbf{v} \in X_{N}, a\left(\mathbf{u}_{N}, \mathbf{v}\right)+b\left(\mathbf{v}, p_{N}\right)=L(\mathbf{v}), \\
\forall q \in M_{N}, b\left(\mathbf{u}_{N}, q\right)=0 .
\end{array}\right.
$$

In order to prove the well-posedness for (3.6), we only need the inf-sup condition in Theorem 2.1, We shall actually prove a uniform inf-sup condition.

\subsection{The discrete inf-sup condition.}

Theorem 3.1. One has on $X_{N} \times M_{N}$ the following uniform inf-sup condition:

$$
\inf _{q \in M_{N}} \sup _{\mathbf{v} \in X_{N}} \frac{|b(\mathbf{v}, q)|}{\|\mathbf{v}\|_{X}\|q\|_{M}}=\frac{1}{\sqrt{3}} .
$$

Proof. As in subsection 2.4 we have

$$
\inf _{q \in M_{N}} \sup _{\mathbf{v} \in X_{N}} \frac{|b(\mathbf{v}, q)|}{\|\mathbf{v}\|_{X}\|q\|_{M}}=\inf _{q \in M_{N}} \frac{\|\mathbf{w}(q)\|_{X}}{\|q\|_{M}}
$$

where, for $q$ in $M_{N}, \mathbf{w}(q)$ is the unique solution to the problem

$$
\mathbf{w} \in X_{N}, \forall \mathbf{v} \in X_{N},(\Delta \mathbf{w}+\operatorname{grad} q, \mathbf{v})_{0, \Omega}=0 .
$$

Using formula (2.56), it is easy to see that, if $q$ belongs to $M_{N}, w_{N}=-(\Delta)^{-1} \operatorname{grad} q$ belongs to $X_{N}$, which terminates the proof.

This result, together with Theorem 2.1 leads to the conclusion:

Theorem 3.2. For any $\mathbf{f}$ in $\mathbf{H}^{-1}(\Omega)$, there exists a unique solution $\left(\mathbf{u}_{N}, p_{N}\right)$ to (3.6) in $X_{N} \times M_{N}$. Furthermore, there exists a positive constant $C$ such that

$$
\forall \mathbf{f} \in \mathbf{H}^{-1}(\Omega), \forall N \geq 2,\left\|\mathbf{u}_{N}\right\|_{X}+\left\|p_{N}\right\|_{M} \leq C\|\mathbf{f}\|_{-1, \Omega} .
$$

3.2. Jacobi polynomials and weighted Sobolev spaces on $[0,1]$. We introduced in (2.34) the weighted Hilbert space $L_{r^{2}}^{2}(] 0,1[)$. More generally, let $\omega$ be a positive function on $[0,1]$ such that for any positive $k, \omega r^{k}$ is integrable on ]0, 1[. We define the space

$$
L_{\omega}^{2}(] 0,1[)=\left\{\varphi \in \mathcal{D}^{\prime}(] 0,1[), \int_{0}^{1} \omega(r)|\varphi|^{2}(r) d r<+\infty\right\} .
$$

It is a Hilbert space when equipped with the natural inner product

$$
(\varphi, \psi)=\int_{0}^{1} \omega \varphi(r) \overline{\psi(r)} d r
$$

and the corresponding norm, denoted by $\|\cdot\|_{\omega}$. For any given weight $\omega$, there exists a sequence of orthogonal polynomials in $L_{w}^{2}(] 0,1[$ ). If $\alpha$ and $\beta$ are two integers and $\omega=\omega_{\alpha, \beta}=(1-r)^{\alpha} r^{\beta}$, it is the sequence of Jacobi polynomials $J_{n}^{\alpha, \beta}$ normalized by $J_{n}^{\alpha, \beta}(1)=\left(\begin{array}{c}n+a \\ n\end{array}\right)$. Their norm is given by

$$
\left\|J_{n}^{\alpha, \beta}\right\|_{\omega_{\alpha, \beta}}^{2}=\frac{(n+\alpha) !(n+\beta) !}{(n+\alpha+\beta) !(2 n+\alpha+\beta+1) n !} .
$$


The unbounded operator $L_{\alpha, \beta}$ on $L_{\omega_{\alpha, \beta}}^{2}(] 0,1[)$ is defined by

$$
L_{\alpha, \beta}=-\frac{1}{\omega_{\alpha, \beta}} \frac{d}{d r}\left[\omega_{\alpha+1, \beta+1} \frac{d}{d r}\right] .
$$

$L_{\alpha, \beta}$ is self-adjoint positive on $L_{\omega_{\alpha, \beta}}^{2}(] 0,1[)$, the eigenfunctions are $J_{n}^{\alpha, \beta}$ with eigenvalues $\lambda_{n}^{\alpha, \beta}=n(n+\alpha+\beta+1)$. The polynomials $J_{n}^{\alpha, \beta}$ satisfy the differential equation

$$
r(1-r)\left(J_{n}^{\alpha, \beta}\right)^{\prime \prime}+(\beta+1-(\alpha+\beta+2) r)\left(J_{n}^{\alpha, \beta}\right)^{\prime}+\lambda_{n}^{\alpha, \beta} J_{n}^{\alpha, \beta}=0 .
$$

Moreover, the Jacobi polynomials are given by the recursion formula

$$
\begin{aligned}
2(n+1) & (n+\alpha+\beta+1)(2 n+\alpha+\beta) J_{n+1}^{\alpha, \beta} \\
= & (2 n+\alpha+\beta+1)\left\{\alpha^{2}-\beta^{2}+2(n+\alpha+\beta+2)(n+\alpha+\beta)(2 r-1)\right\} J_{n}^{\alpha, \beta} \\
& -2(n+\alpha)(n+\beta)(2 n+\alpha+\beta+2) J_{n-1}^{\alpha, \beta} \\
J_{0}^{\alpha, \beta}= & 1 ; J_{1}^{\alpha, \beta}=(\alpha+\beta+2) r-(\beta+1) .
\end{aligned}
$$

For any positive integer $\tau$, we denote by $H_{r^{2}}^{\tau}(] 0,1[)$ the space of distributions defined on ]0,1[ whose derivatives up to order $\tau$ belong to $L_{r^{2}}^{2}(] 0,1[$ ), furnished with the norm defined by $\|\psi\|_{\tau, r}^{2}=\sum_{j=0}^{\tau}\|\psi\|_{r}^{2}$.

Theorem 3.3. 1. For any $q$ in $L_{r^{2}}^{2}(] 0,1[)$, there exists a unique polynomial $\pi_{r, N} q$ in $\mathbb{P}_{N}([0,1])$ such that

$$
\forall Q \in \mathbb{P}_{N}([0,1]),\left\|q-\pi_{r, N} q\right\|_{r} \leq\|q-Q\|_{r} .
$$

2. For any positive integer $\tau$ one has

$$
\forall q \in H_{r^{2}}^{\tau}(] 0,1[),\left\|q-\pi_{r, N} q\right\|_{r} \leq C N^{-\tau}\|q\|_{p, r} .
$$

Proof. The proof is now classical and will be omitted here. Results of the same type have been obtained in [1] for the weight $\omega_{\alpha, \alpha}$ and in [9] for the weight $r$. It follows the same path as in the proof of Theorem [3.4. It relies on the expansion of $q$ on the Jacobi polynomials $J_{n+1}^{\alpha, \beta}$ (here $\alpha=0$ and $\beta=2$ ) and multiple use of the Green formula.

3.3. Approximation results. Due to the ellipticity of the sesquilinear form $a$ and the uniform inf-sup condition, Theorem 1.1 in [7] gives a first approximation result: there exists a positive constant $C$ such that, for any $N \geq 2$, if $(\mathbf{u}, p)$ and $\left(\mathbf{u}_{N}, p_{N}\right)$ are the solutions to (2.3) and (3.6), respectively,

$$
\left\|\mathbf{u}-\mathbf{u}_{N}\right\|_{X}+\left\|p-p_{N}\right\|_{M} \leq C\left(\inf _{v_{N} \in X_{N}}\left\|\mathbf{u}-\mathbf{v}_{N}\right\|_{X}+\inf _{q_{N} \in M_{N}}\left\|p-q_{N}\right\|_{M}\right),
$$

where $V_{N}$ is the discrete space corresponding to $V$ :

$$
V_{N}=\left\{\mathbf{v}_{N} \in X_{N}, \forall q_{N} \in M_{N}, b\left(\mathbf{v}_{N}, q_{N}\right)=0\right\} .
$$

It remains to estimate the expressions in the right-hand side of (3.18).

Theorem 3.4. Let $\tau$ be a positive integer. For any $q$ in $M \cap H^{\tau}(\Omega)$, there exists a unique $\tilde{q}$ projection of $q$ on $M_{K, L}$ in $L^{2}(\Omega)$. Moreover, $\tilde{q}$ belongs to $M$ and

$$
\|q-\tilde{q}\|_{M} \leq C\left(K^{-\tau}+L^{-\tau}\right)\|q\|_{\tau, \Omega} .
$$


Proof. We denote by $\pi_{S, K}$ the projection in $L^{2}(S)$ on the $K$ first eigenspaces, i.e., on $E_{K}=\bigoplus_{m=0}^{K-1} \mathcal{L}\left(Y_{m,-m}, \ldots, Y_{m, m}\right)$. If

$$
\eta=\sum_{m=0}^{+\infty} \sum_{l=-m}^{m} \eta_{m, l} Y_{m, l}
$$

then

$$
\pi_{S, K} \eta=\sum_{m=0}^{K-1} \sum_{l=-m}^{m} \eta_{m, l} Y_{m, l} .
$$

Using the fact that the $Y_{m, l}$ are the eigenfunctions for the eigenvalues $\lambda_{m}=$ $m(m+1)$, we get for any integer $n$

$$
\eta_{m, l}=\left(\eta, Y_{m, l}\right)_{0, S}=\frac{1}{\lambda_{m}^{n}}\left(\left(-\Delta_{S}\right)^{n} \eta, Y_{m, l}\right)_{0, S}
$$

So that

$$
\left\|\eta-\pi_{S, K} \eta\right\|_{0, S} \leq \frac{1}{\lambda_{K}^{n}}\left\|\left(-\Delta_{S}\right)^{n} \eta\right\|_{0, S}
$$

and

$$
\left\|\eta-\pi_{S, K} \eta\right\|_{0, S} \leq \frac{1}{\lambda_{K}^{n+\frac{1}{2}}}\left\|\left(-\Delta_{S}\right)^{n+\frac{1}{2}} \eta\right\|_{0, S} .
$$

It is well known (see [12]) that for any real number $\sigma,\left\|\left(-\Delta_{S}\right)^{\sigma} \eta\right\|_{0, S} \leq\|\eta\|_{2 \sigma, S}$, so that, for any integer $n$, if $\eta$ belongs to $H^{n}(S)$.

$$
\left\|\eta-\pi_{S, K} \eta\right\|_{0, S} \leq \frac{1}{\lambda_{K}^{\frac{n}{2}}}\|\eta\|_{n, S} \leq \frac{1}{K^{n}}\|\eta\|_{n, S} .
$$

Now $\tilde{q}$ is given by

$$
\tilde{q}=\pi_{r, L} \pi_{S, K} q=\sum_{m=0}^{K-1} \sum_{l=-m}^{m}\left(\pi_{r, L} q_{m, l}\right) Y_{m, l}
$$

and, using Theorem 3.3 the proof is now complete.

Theorem 3.5. Let $\tau$ be a positive integer. For any $\mathbf{v}$ in $V \cap H^{\tau}(\Omega)$, there exists $\mathbf{v}_{N}$ in $X_{N} \cap V$ such that

$$
\left\|\mathbf{v}-\mathbf{v}_{N}\right\|_{X} \leq C N^{1-\tau}\|\mathbf{v}\|_{\tau, \Omega} .
$$

Proof. We first identify the space $V$. Recall that it is defined by

$$
V=\left\{\mathbf{v} \in X, \forall q \in M,(\operatorname{div} \mathbf{v}, q)_{0, \Omega}=0\right\} .
$$

Any $q$ in $M$ is written as

$$
q=\sum_{m=0}^{+\infty} \sum_{l=-m}^{m} q_{m, l} Y_{m, l}, \quad q_{m, l} \in L_{r^{2}}^{2}(] 0,1[),\left(q_{0,0}, 1\right)_{r}=0 .
$$

Let $\mathbf{v}$ be written as in (2.37), i.e.,

$$
\mathbf{v}=\sum_{m=1}^{+\infty} \sum_{l=-m}^{m} t_{m, l} \mathbf{T}_{m, l}+\sum_{m=0}^{+\infty} \sum_{l=-m-1}^{m+1} v_{m, l} \mathbf{V}_{m, l}+\sum_{m=1}^{+\infty} \sum_{l=-m+1}^{m-1} w_{m, l} \mathbf{W}_{m, l} .
$$


We can calculate the divergence of $\mathbf{v}$ as

$$
\operatorname{div} \mathbf{v}=\sum_{m=1}^{+\infty} \sum_{l=-m}^{m}\left(m \varphi_{m-1, l}+(m+1) \psi_{m+1, l}\right) Y_{m, l}+\psi_{1,0} Y_{1,0}
$$

where the $\varphi_{m, l}$ and $\psi_{m, l}$ have been defined in (2.41). Taking into account the boundary conditions, we deduce that $\mathbf{v}$ belongs to $V$ if and only if

$$
\left\{\begin{array}{l}
\forall m \geq 1, m \varphi_{m-1, l}+(m+1) \psi_{m+1, l}=0 \\
w_{1,0}=0
\end{array}\right.
$$

The functions

$$
\begin{gathered}
\psi_{m, l}=r^{-(m+1)} \frac{d}{d r}\left(r^{(m+1)} w_{m, l}\right), 2 \leq m \leq N, \\
\tau_{m, l}=r^{-(m+1)} \frac{d}{d r}\left(r^{(m+1)} t_{m, l}\right), 1 \leq m \leq N,
\end{gathered}
$$

belong to $L_{r^{2}}^{2}$, and we can define $\tilde{\psi}_{m, l}=\pi_{r, N-1} \psi_{m, l}$ and $\tilde{\tau}_{m, l}=\pi_{r, N-1} \tau_{m, l}$.

We look now for $\tilde{w}_{m, l}$ and $\tilde{t}_{m, l}$ in $\tilde{\mathbb{P}}_{N}([0,1])$ such that

$$
\begin{aligned}
r^{-(m+1)} \frac{d}{d r}\left(r^{(m+1)} \tilde{w}_{m, l}\right) & =\tilde{\psi}_{m, l}, \\
r^{-(m+1)} \frac{d}{d r}\left(r^{(m+1)} \tilde{t}_{m, l}\right) & =\tilde{\tau}_{m, l} .
\end{aligned}
$$

Due to the orthogonality conditions in $L_{r^{2}}^{2}, \tilde{w}_{m, l}$ and $\tilde{t}_{m, l}$ can be defined by

$$
\begin{gathered}
\tilde{w}_{m, l}=r^{-(m+1)} \int_{0}^{r} \varrho^{m+1} \tilde{\psi}_{m, l}(\varrho) d \varrho, 2 \leq m \leq N, \\
\tilde{t}_{m, l}=r^{-(m+1)} \int_{0}^{r} \varrho^{m+1} \tilde{\tau}_{m, l}(\varrho) d \varrho, 1 \leq m \leq N .
\end{gathered}
$$

Choosing $\tilde{w}_{1,0}=0$, and, for $0 \leq m \leq N-2$,

$$
\tilde{\varphi}_{m, l}=-\frac{m+2}{m+1} \tilde{\psi}_{m+2, l}
$$

defines a function in $\mathbb{P}_{N-1}([0,1])$, which coincides with $\pi_{r, N-1} \varphi_{m, l}$. We now define $\tilde{v}_{m, l}$ as

$$
\tilde{v}_{m, l}=-r^{m} \int_{r}^{1} \varrho^{-m} \tilde{\varphi}_{m, l}(\varrho) d \varrho, 0 \leq m \leq N-2,
$$

which belongs to $\tilde{\mathbb{P}}_{N}([0,1]) \oplus \mathbb{Q}_{m}$.

Function $\mathbf{v}_{N}$ is finally given by

$$
\mathbf{v}_{N}=\sum_{m=1}^{+\infty} \sum_{l=-m}^{m} \tilde{t}_{m, l} \mathbf{T}_{m, l}+\sum_{m=0}^{+\infty} \sum_{l=-m-1}^{m+1} \tilde{v}_{m, l} \mathbf{V}_{m, l}+\sum_{m=1}^{+\infty} \sum_{l=-m+1}^{m-1} \tilde{w}_{m, l} \mathbf{W}_{m, l} .
$$

By construction it belongs to $V \cap X_{N}$, and, by Theorem 3.4 and its proof, the estimate follows.

Theorems 3.4 and 3.5 together with (3.18) give the final optimal error estimate:

Theorem 3.6. Let $\tau$ be a positive integer. If $\mathbf{f}$ belongs to $\mathbf{H}^{\tau}(\Omega)$, the solutions $(\mathbf{u}, p)$ and $\left(\mathbf{u}_{N}, p_{N}\right)$ to (2.3) and (3.6) satisfy the estimate

$$
\left\|\mathbf{u}-\mathbf{u}_{N}\right\|_{X}+\left\|p-p_{N}\right\|_{M} \leq C N^{-1-\tau}\|\mathbf{f}\|_{p, \Omega} .
$$




\section{Coupling SPECTRAL METHOD AND TRANSPARENT BOUNDARY CONDITION}

In order to settle a variational formulation to problems in unbounded domains, weighted Sobolev spaces have been introduced in [10]. We recall here the definitions and some useful properties. For a smooth unbounded domain $\Omega$, we introduce the space

$$
W^{1}(\Omega)=\left\{v \in \mathcal{D}^{\prime}(\Omega), \operatorname{grad} v \in L^{2}(\Omega),\left(1+r^{2}\right)^{-\frac{1}{2}} v \in L^{2}(\Omega)\right\} .
$$

It is a Hilbert space, furnished with the natural inner product and corresponding norm

$$
\|v\|_{W^{1}(\Omega)}^{2}=\left\|\left(1+r^{2}\right)^{-\frac{1}{2}} v\right\|_{0, \Omega}^{2}+\|\operatorname{grad} v\|_{0, \Omega}^{2} .
$$

The properties of such spaces can be found for instance in [6]. The notations are extended to vectors by boldfacing. Consider first the Stokes problem in the whole space

$$
\begin{cases}-\Delta \mathbf{u}+\operatorname{grad} p=\mathbf{f} & \text { in } \mathbb{R}^{3}, \\ \operatorname{div} \mathbf{u}=0 & \text { in } \mathbb{R}^{3} .\end{cases}
$$

The data $\mathbf{f}$ is assumed to be compactly supported in the ball $B(R)$ of radius $R$. Following [6], if $f$ is compactly supported and belongs to $\mathbf{L}^{2}\left(\mathbb{R}^{3}\right)$, problem (4.3) has a unique weak solution $(\mathbf{u}, p)$ in $\mathbf{W}^{1}\left(\mathbb{R}^{3}\right) \times L^{2}\left(\mathbb{R}^{3}\right)$.

Remark 4.1. In the two-dimensional case, $\mathbb{R}$ belongs to $W^{1}(\Omega)$, an orthogonality condition is needed on $f$, and the pressure $p$ is defined modulo a constant.

In order to compute $(\mathbf{u}, p)$, we shall introduce a fictitious boundary, the sphere of radius $R$, and solve the Stokes problem in the ball $B(R)$ with the so-called transparent boundary condition. This boundary condition accounts for the solution outside $B(R)$.

4.1. Transparent boundary condition and reduction to a bounded domain. Following, we shall denote $\Omega=B(R), \Omega^{\prime}=\mathbb{R}^{3}-\Omega, S_{R}$ their common boundary. The unit normal vector to $S_{R}$ is oriented toward the exterior of $\Omega$, and we shall call it $\mathbf{n}$ (it is $\mathbf{e}_{r}$ in the notation of subsection 2.3). Problem (4.3) is equivalent to the coupling

$$
\left\{\begin{array} { l l } 
{ - \Delta \mathbf { u } _ { 1 } + \operatorname { g r a d } p _ { 1 } = f } & { \text { in } \Omega , } \\
{ \operatorname { d i v } \mathbf { u } _ { 1 } = 0 } & { \text { in } \Omega , }
\end{array} \left\{\begin{array}{ll}
-\Delta \mathbf{u}_{2}+\operatorname{grad} p_{2}=0 & \text { in } \Omega^{\prime}, \\
\operatorname{div} \mathbf{u}_{2}=0 & \text { in } \Omega^{\prime},
\end{array}\right.\right.
$$

with the transmission conditions

$$
\begin{cases}\mathbf{u}_{1}=\mathbf{u}_{2} & \text { on } S_{R} \\ \sigma_{\mathbf{n}}\left(\mathbf{u}_{1}\right)=\sigma_{\mathbf{n}}\left(\mathbf{u}_{2}\right) & \text { on } S_{R}\end{cases}
$$

where $\sigma_{\mathbf{n}}$ is the normal strain, i.e.,

$$
\sigma_{\mathbf{n}}(\mathbf{u})=\frac{\partial \mathbf{u}}{\partial \mathbf{n}}-p \mathbf{n}
$$

Consider the problem

$$
\begin{cases}-\Delta \mathbf{w}+\operatorname{grad} q=0 & \text { in } \Omega^{\prime}, \\ \operatorname{div} \mathbf{w}=0 & \text { in } \Omega^{\prime}, \\ \mathbf{w}=\mathbf{g} & \text { on } S_{R}\end{cases}
$$


This problem is well posed, due to the key properties of this weighted space, i.e., a Poincare inequality. Let $W_{0}^{1}\left(\Omega^{\prime}\right)$ be the closure of $\mathcal{D}\left(\Omega^{\prime}\right)$ in $W^{1}\left(\Omega^{\prime}\right)$. It is also the subspace of functions in $W^{1}\left(\Omega^{\prime}\right)$ whose trace on $S_{R}$ vanishes. The semi-norm $\|\operatorname{grad} .\|_{0, \Omega}$ is on $W_{0}^{1}(\Omega)$ a norm, equivalent to the $\|\cdot\|_{W^{1}\left(\Omega^{\prime}\right)}$ norm.

Theorem 4.1 ([8]). For any $g$ in $\mathbf{H}^{\frac{1}{2}}\left(S_{R}\right)$, there exists a unique weak solution $(\mathbf{w}, q)$ to (4.7) in $\mathbf{W}^{1}\left(\Omega^{\prime}\right) \times L^{2}\left(\Omega^{\prime}\right)$. Moreover, there exists a positive real number $C$ such that, for any $g$,

$$
\|\mathbf{w}\|_{W^{1}\left(\Omega^{\prime}\right)} \leq C\|\mathbf{g}\|_{\frac{1}{2}, S_{R}} .
$$

Denote by $\mathcal{K}$ the linear operator defined on $\mathbf{H}^{\frac{1}{2}}\left(S_{R}\right)$ by

$$
\mathcal{K} \mathbf{g}=-\sigma_{\mathbf{n}}(\mathbf{w}), \mathbf{w} \text { solution to }(4.7) .
$$

Using the transmission conditions on $S_{R}$, problem (4.3) is equivalent to the following boundary value problem in the bounded domain $\Omega$ :

$$
\begin{cases}-\Delta \mathbf{u}+\operatorname{grad} p=\mathbf{f} & \text { in } \Omega \\ \operatorname{div} \mathbf{u}=0 & \text { in } \Omega \\ \sigma_{\mathbf{n}}(\mathbf{u})+\mathcal{K} \mathbf{u}=0 & \text { on } S_{R}\end{cases}
$$

We shall now give an explicit form for $\mathcal{K}$ and some useful properties.

4.2. Basic properties and expression of the transparent operator $K$. We defined in subsection 2.3 Sobolev spaces of fractional order on the sphere $S=S_{1}$ for scalar functions. We introduce now Sobolev spaces of fractional order on $S_{R}$ for vector-valued functions: for any real number $s, \mathbf{H}^{s}\left(S_{R}\right)$ is the space of functions $\mathbf{g}$ in $\left(\mathcal{D}^{\prime}\left(S_{R}\right)\right)^{3}$ given by

$$
\mathbf{g}=\sum_{m=1}^{+\infty} \sum_{l=-m}^{m} g_{m, l}^{T} \mathbf{T}_{m, l}+\sum_{m=0}^{+\infty} \sum_{l=-m-1}^{m+1} g_{m, l}^{V} \mathbf{V}_{m, l}+\sum_{m=1}^{+\infty} \sum_{l=-m+1}^{m-1} g_{m, l}^{W} \mathbf{W}_{m, l}
$$

and such that

$$
\begin{aligned}
\sum_{m=1}^{+\infty} & \sum_{l=-m}^{m} m(m+1)(m+1)^{2 s}\left|g_{m, l}^{T}\right|^{2} \\
& +\sum_{m=0}^{+\infty} \sum_{l=-m-1}^{m+1}(m+1)(2 m+3)\left(\frac{3(m+1)^{2}}{m+2}\right)^{2 s}\left|g_{m, l}^{V}\right|^{2} \\
& +\sum_{m=1}^{+\infty} \sum_{l=-m+1}^{m-1} m(2 m-1)(m+1)^{2 s}\left|g_{m, l}^{W}\right|^{2}<+\infty
\end{aligned}
$$

The norm of $\mathbf{g}$ in $\mathbf{H}^{s}\left(S_{R}\right)$ is given by

$$
\begin{aligned}
\|\mathbf{g}\|_{s, S_{R}}^{2}= & \sum_{m=1}^{+\infty} \sum_{l=-m}^{m} m(m+1)(m+1)^{2 s}\left|g_{m, l}^{T}\right|^{2} \\
& +\sum_{m=0}^{+\infty} \sum_{l=-m-1}^{m+1}(m+1)(2 m+3)\left(\frac{3(m+1)^{2}}{m+2}\right)^{2 s}\left|g_{m, l}^{V}\right|^{2} \\
& +\sum_{m=1}^{+\infty} \sum_{l=-m+1}^{m-1} m(2 m-1)(m+1)^{2 s}\left|g_{m, l}^{W}\right|^{2}
\end{aligned}
$$


We add a last definition: the duality between $\mathbf{H}^{\frac{1}{2}}\left(S_{R}\right)$ and $\mathbf{H}^{-\frac{1}{2}}\left(S_{R}\right)$. If $\mathbf{g}$ in $\mathbf{H}^{\frac{1}{2}}\left(S_{R}\right)$, and $\mathbf{h}$ in $\mathbf{H}^{-\frac{1}{2}}\left(S_{R}\right)$ are given by

$$
\begin{aligned}
& \mathbf{g}=\sum_{m=1}^{+\infty} \sum_{l=-m}^{m} g_{m, l}^{T} \mathbf{T}_{m, l}+\sum_{m=0}^{+\infty} \sum_{l=-m-1}^{m+1} g_{m, l}^{V} \mathbf{V}_{m, l}+\sum_{m=1}^{+\infty} \sum_{l=-m+1}^{m-1} g_{m, l}^{W} \mathbf{W}_{m, l}, \\
& \mathbf{h}=\sum_{m=1}^{+\infty} \sum_{l=-m}^{m} h_{m, l}^{T} \mathbf{T}_{m, l}+\sum_{m=0}^{+\infty} \sum_{l=-m-1}^{m+1} h_{m, l}^{V} \mathbf{V}_{m, l}+\sum_{m=1}^{+\infty} \sum_{l=-m+1}^{m-1} h_{m, l}^{W} \mathbf{W}_{m, l},
\end{aligned}
$$

then

$$
\begin{aligned}
\langle\mathbf{g}, \mathbf{h}\rangle_{S_{R}}= & \sum_{m=1}^{+\infty} \sum_{l=-m}^{m} m(m+1) g_{m, l}^{T} \overline{h_{m, l}^{T}} \\
& +\sum_{m=0}^{+\infty} \sum_{l=-m-1}^{m+1}(m+1)(2 m+3) g_{m, l}^{V} \overline{h_{m, l}^{V}} \\
& +\sum_{m=1}^{+\infty} \sum_{l=-m+1}^{m-1} m(2 m-1) g_{m, l}^{W} \overline{h_{m, l}^{W}} .
\end{aligned}
$$

Theorem 4.2. $\mathcal{K}$ is a linear continuous operator from $\mathbf{H}^{\frac{1}{2}}\left(S_{R}\right)$ to $\mathbf{H}^{-\frac{1}{2}}\left(S_{R}\right)$. It is self-adjoint, and

$$
\langle\mathcal{K} \mathbf{g}, \mathbf{g}\rangle_{S_{R}}=\|\mathbf{g}\|_{\frac{1}{2}, S_{R}}^{2} .
$$

If $\mathbf{g}$ is given by (4.11), then $\mathcal{K} \mathbf{g}$ is given by

$$
\begin{aligned}
\mathcal{K} \mathbf{g}=\frac{1}{R}\left(\sum_{m=1}^{+\infty} \sum_{l=-m}^{m}(m+1) g_{m, l}^{T} \mathbf{T}_{m, l}\right. & +\sum_{m=0}^{+\infty} \sum_{l=-m-1}^{m+1} \frac{3(m+1)^{2}}{m+2} g_{m, l}^{V} \mathbf{V}_{m, l} \\
& \left.+\sum_{m=1}^{+\infty} \sum_{l=-m+1}^{m-1}(m+1) g_{m, l}^{W} \mathbf{W}_{m, l}\right) .
\end{aligned}
$$

Proof. The proof of formula (4.16) lies in the explicit solution of (4.7) in four steps.

1. Note first that the pressure $q$ is harmonic in $\Omega^{\prime}$ and belongs to $L^{2}\left(\Omega^{\prime}\right)$. Then it is given by

$$
q=\sum_{m=0}^{+\infty} \sum_{l=-m}^{m} q_{m, l}(r) Y_{m, l}
$$

with $q_{m, l}(r)=\alpha_{m, l} r^{-(m+1)}$ for $m \geq 1$ and $q_{0,0}=0$.

2. With the notation of subsection 2.3 ,

$$
\mathbf{w}=\sum_{m=1}^{+\infty} \sum_{l=-m}^{m} t_{m, l} \mathbf{T}_{m, l}+\sum_{m=0}^{+\infty} \sum_{l=-m-1}^{m+1} v_{m, l} \mathbf{V}_{m, l}+\sum_{m=1}^{+\infty} \sum_{l=-m+1}^{m-1} w_{m, l} \mathbf{W}_{m, l}
$$

We can calculate the divergence of $\mathbf{w}$ as

$$
\operatorname{div} \mathbf{w}=\sum_{m=1}^{+\infty} \sum_{l=-m}^{m}\left(m \varphi_{m-1, l}+(m+1) \psi_{m+1, l}\right) Y_{m, l}+\psi_{1,0} Y_{0,0}
$$


where the $\varphi_{m, l}$ and $\psi_{m, l}$ have been defined in (2.41). Then the divergence-free condition resumes to

$$
\left\{\begin{array}{l}
\forall m \geq 1, m \varphi_{m-1, \ell}+(m+1) \psi_{m+1, l}=0 \\
\psi_{1,0}=0
\end{array}\right.
$$

3. The first equation in (4.7) is expanded as in subsection 2.3.

$$
\forall m \geq 1, \forall l,-m \leq l \leq m,\left(\Delta_{r}-\frac{m(m+1)}{r^{2}}\right) t_{m, l}=0,
$$

$$
\forall m \geq 0, \forall l,-m-1 \leq l \leq m+1, \frac{d}{d r}\left(r^{m+2}\left(\varphi_{m, l}-\frac{1}{2 m+3} p_{m+1, l}\right)\right)=0,
$$

$$
\forall m \geq 1, \forall l,-m+1 \leq l \leq m-1, \frac{d}{d r}\left(r^{-(m-1)}\left(\psi_{m, l}-\frac{1}{2 m-1} p_{m-1, l}\right)\right)=0 .
$$

Equation (4.18) is solved explicitly as $t_{m, l}=g_{m, l}^{T}\left(\frac{r}{R}\right)^{-(m+1)}$. As for (4.19) and (4.20), we use the divergence-free condition to get

$$
\forall m \geq 0, \varphi_{m, l}=-\frac{m+2}{2 m+3} q_{m+1, l}
$$

and

$$
\forall m \geq 0, \alpha_{m+1, l}=R^{m+1} \frac{(m+1)(2 m+3)(2 m+1)}{m+2} g_{m, l}^{V} .
$$

4. The operator $\mathcal{K}$ can be expressed on the basis as

$$
\begin{aligned}
\mathcal{K} \mathbf{g} & =\sum_{m=1}^{+\infty} \sum_{l=-m}^{m} \tau_{m, l} \mathbf{T}_{m, l}+\sum_{m=0}^{+\infty} \sum_{l=-m-1}^{m+1}\left(\varphi_{m, l}-\frac{1}{2 m+3} q_{m+1, l}\right) \mathbf{V}_{m, l} \\
& +\sum_{m=1}^{+\infty} \sum_{l=-m+1}^{m-1}\left(\psi_{m, l}-\frac{1}{2 m-1} q_{m-1, l}\right) \mathbf{W}_{m, l}-\frac{1}{R} \sum_{m=1}^{+\infty} \sum_{l=-m}^{m}(m+1) g_{m, l}^{T} \mathbf{T}_{m, l} \\
& +\frac{1}{R} \sum_{m=0}^{+\infty} \sum_{l=-m-1}^{m+1} m g_{m, l}^{V} \mathbf{V}_{m, l}-\frac{1}{R} \sum_{m=1}^{+\infty} \sum_{l=-m+1}^{m-1}(m+1) g_{m, l}^{W} \mathbf{W}_{m, l}
\end{aligned}
$$

and formula (4.16) follows. As for the properties of $\mathcal{K}$, they follow in a straightforward way from (4.16) and the definitions of the spaces.

4.3. The variational formulation. For simplicity we choose $R=1$. The constants for a general $R$ are easily obtained by scaling. We shall now write a variational formulation for problem (4.10) in $\Omega=B(1)$. We define on $H^{1}(\Omega)$ a sesquilinear form by

$$
a_{R}(\mathbf{u}, \mathbf{v})=a(\mathbf{u}, \mathbf{v})+\langle\mathcal{K} \mathbf{u}, \mathbf{v}\rangle_{S_{R}} .
$$

Lemma 4.1. The sesquilinear form $a_{R}$ defines on $\mathbf{H}^{1}(\Omega)$ an inner product. The corresponding norm is equivalent to the natural norm in $\mathbf{H}^{1}(\Omega)$. 
The proof is straightforward, due to the properties of $\mathcal{K}$. The Hilbert space $X$ is now $\mathbf{H}^{1}(\Omega)$, furnished with the sesquilinear form $a_{R}$, and $M=\mathbf{L}^{2}(\Omega)$. The variational formulation reads

$$
\left\{\begin{array}{l}
\forall \mathbf{v} \in X, a_{R}(\mathbf{u}, \mathbf{v})+b(\mathbf{v}, p)=L(\mathbf{v}), \\
\forall q \in M, b(\mathbf{u}, q)=0 .
\end{array}\right.
$$

Theorem 4.3. Problem (4.5) has a unique solution. In particular the inf-sup condition is fulfilled as

$$
\inf _{q \in M} \sup _{\mathbf{v} \in X} \frac{|b(\mathbf{v}, q)|}{\|\mathbf{v}\|_{X}\|q\|_{M}}=1 .
$$

Proof. By Theorem 2.1 it is enough to prove (4.26). We proceed as in subsection 2.4 :

$$
\inf _{q \in M} \sup _{\mathbf{v} \in X} \frac{|b(\mathbf{v}, q)|}{\|\mathbf{v}\|_{X}\|q\|_{M}}=\inf _{q \in M} \frac{\|\mathbf{w}(q)\|_{X}}{\|q\|_{M}},
$$

where, for $q$ in $M, \mathbf{w}(q)$ is the unique solution to the problem

$$
\mathbf{w} \in X, \forall \mathbf{v} \in X, a_{R}(\mathbf{w}, \mathbf{v})=b(\mathbf{v}, q),
$$

or equivalently,

$$
\begin{cases}\mathbf{w} \in X=\mathbf{H}^{1}(\Omega), & \\ \Delta \mathbf{w}+\operatorname{grad} q=\mathbf{0} & \text { in } \Omega, \\ \frac{\partial \mathbf{w}}{\partial r}+q \mathbf{e}_{r}+\mathcal{K} \mathbf{w}=\mathbf{0} & \text { on } S_{R} .\end{cases}
$$

We now expand $q$ as in (2.33) and $\mathbf{w}$ as in (2.37), use the boundary condition, and obtain the result.

4.4. A Galerkin method. For $N \geq 2$, the discrete spaces are the same as in Section 3 and the discrete problem reads, find $\left(\mathbf{u}_{N}, p_{N}\right)$ in $X_{N} \times M_{N}$ such that

$$
\left\{\begin{array}{l}
\forall \mathbf{v} \in X_{N}, a_{R}\left(\mathbf{u}_{N}, \mathbf{v}\right)+b\left(\mathbf{v}, p_{N}\right)=L(\mathbf{v}), \\
\forall q \in M_{N}, b\left(\mathbf{u}_{N}, q\right)=0 .
\end{array}\right.
$$

Theorem 4.4. One has on $X_{N} \times M_{N}$ the following uniform inf-sup condition:

$$
\inf _{q \in M_{N}} \sup _{\mathbf{v} \in X_{N}} \frac{|b(\mathbf{v}, q)|}{\|\mathbf{v}\|_{X}\|q\|_{M}}=1 .
$$

Proof. As in the previous section we have

$$
\inf _{q \in M_{N}} \sup _{\mathbf{v} \in X_{N}} \frac{|b(\mathbf{v}, q)|}{\|\mathbf{v}\|_{X}\|q\|_{M}}=\inf _{q \in M_{N}} \frac{\|\mathbf{w}(q)\|_{X}}{\|q\|_{M}}
$$

where, for $q$ in $M_{N}, \mathbf{w}(q)$ is the unique solution to the problem

$$
\mathbf{w} \in X_{N}, \forall \mathbf{v} \in X_{N},(\Delta \mathbf{w}+\operatorname{grad} q, \mathbf{v})_{0, \Omega}+\left\langle\mathcal{K} \mathbf{w}+\frac{\partial \mathbf{w}}{\partial r}+q \mathbf{e}_{r}, \mathbf{v}\right\rangle_{S_{R}}=0
$$

Using formula (2.56), it is easy to see that, if $q$ belongs to $M_{N}, w_{N}=-(\Delta)^{-1} \operatorname{grad} q$ belongs to $X_{N}$, which ends the proof.

This result, together with Theorem 2.1 leads to the conclusion: 
Theorem 4.5. For any $\mathbf{f}$ in $\mathbf{H}^{-1}(\Omega)$, there exists a unique solution $\left(\mathbf{u}_{N}, p_{N}\right)$ to (4.30) in $X_{N} \times M_{N}$. Furthermore, there exists a positive real number $C$ such that

$$
\forall \mathbf{f} \in \mathbf{H}^{-1}(\Omega), \forall N \geq 2,\left\|\mathbf{u}_{N}\right\|_{X}+\left\|p_{N}\right\|_{M} \leq C\|\mathbf{f}\|_{-1, \Omega} .
$$

Slight modifications to the proofs in subsection 3.3 give the optimal error estimates:

Theorem 4.6. Let $p$ be a positive integer. If $\mathbf{f}$ belongs to $\mathbf{H}^{p}(\Omega)$, the solutions $(\mathbf{u}, p)$ and $\left(\mathbf{u}_{N}, p_{N}\right)$ to (4.25) and (4.30) satisfy the estimate

$$
\left\|\mathbf{u}-\mathbf{u}_{N}\right\|_{X}+\left\|p-p_{N}\right\|_{M} \leq C N^{-1-p}\|\mathbf{f}\|_{p, \Omega} .
$$

\section{Conclusion}

We have introduced here a spectral method for an academic problem, which has the important feature of showing no parasitic modes for pressure. For applications, one still needs to introduce a collocation method. This can be done in the same fashion as in 9. It will then be based on fast computations of spherical harmonics (see [5]). In more general geometries, it can be used through domain decomposition.

\section{REFERENCES}

1. C. Bernardi and Y. Maday, Approximations spectrales de problèmes aux limites elliptiques, Mathématiques et Applications, vol. 10, Springer-Verlag, Paris, 1992. MR 94f:65112

2. C. Canuto, S. I. Hariharan and L. Lustman, Spectral methods for exterior elliptic problems, Numer. Math. 46 (1985), 505-520. MR 86j:65148

3. C. Cohen-Tannoudji, B. Diu and F. Laloë, Mécanique quantique, Hermann, 1977.

4. E. Darve, Fast multiple method: a mathematical study, C. R. Acad. Sci. Paris Ser. I Math. 325 (1997), 1037-1042. MR 98m:65208

5. J. R. Driscoll and D. M. Healy, Jr., Computing Fourier transforms and convolutions on the 2-sphere, Advances in Applied Mathematics 15 (1994), 202-250. MR 95h:65108

6. V. Girault, The Stokes problem and vector potential operator in three-dimensional exterior domains. An approach in weighted Sobolev spaces, Differential Integral Equations 7 (1994), 535-570. MR 94j:35125

7. V. Girault and P.-A. Raviart, Finite element methods for Navier-Stokes equations. Theory and algorithms, Springer Series in Computational Mathematics, 5, Springer-Verlag, Berlin, 1986. MR 88b:65129

8. V. Girault and A. Sequeira, A well-posed problem for the exterior Stokes equations in two and three dimensions, Arch. Rational. Mech. Anal. 114 (1991), 313-333. MR 92c:35093

9. L. Halpern, Spectral methods in polar coordinates for the Stokes problem. Application to computation in unbounded domains, Math. Comp. 65, no. 214 (1996), 507-531. MR 97e:65100

10. B. Hanouzet, Espaces de Sobolev avec poids. Application au problème de Dirichlet dans un demi-espace, Rend. Sem. Mat. Univ. Padova 46 (1971), 227-272. MR 46:9517

11. M. Lenoir and A. Tounsi, The localized finite element method and its application to the twodimensional sea-keeping problem, SIAM J. Numer. Anal. 25 (1988), 729-752. MR 89k:65138

12. J.-L. Lions and E. Magenes, Problèmes aux limites non homogènes et applications. Dunod, 1968. MR 40:512 MR 40:513

13. B. Mercier and G. Raugel, Résolution d'un problème aux limites dans un ouvert axisymétrique par éléments finis en $r, z$ et séries de Fourier en $\theta$, RAIRO Anal. Numér. 16 (1982), 405-471. MR 84g:65154

14. J.-C. Nedelec, Résolution des Equations de Maxwell par Méthodes Intégrales, Cours de DEA de l'Ecole Polytechnique, 1998.

15. V. Rokhlin, Solution of acoustic scattering problems by means of second kind integral equations Wave Motion 5 (1983), 257-272. MR 85a:76087

LaGa, Institut Galilée, Université Paris 13, 93430 Villetaneuse, France

E-mail address: halpern@yap.math.univ-paris13.fr 